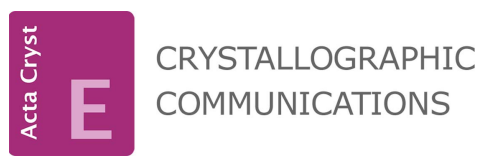

ISSN 2056-9890

Received 29 June 2015

Accepted 2 July 2015

Edited by M. Zeller, Youngstown State University, USA

Keywords: crystal structure; $\pi-\pi$ stacking; hydrogen bond; halogen bond; halogenhalogen interaction

CCDC reference: 1410048 Supporting information: this article has supporting information at journals.iucr.org/e

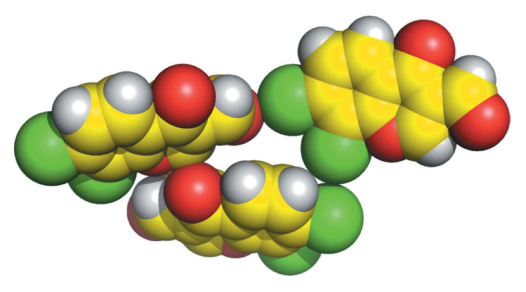

OPEN $\odot$ ACCESS

\section{Crystal structure of 7,8-dichloro-4-oxo-4H-chro- mene-3-carbaldehyde}

\author{
Yoshinobu Ishikawa
}

School of Pharmaceutical Sciences, University of Shizuoka, 52-1 Yada, Suruga-ku, Shizuoka 422-8526, Japan. *Correspondence e-mail: ishi206@u-shizuoka-ken.ac.jp

In the title compound, $\mathrm{C}_{10} \mathrm{H}_{4} \mathrm{Cl}_{2} \mathrm{O}_{3}$, a dichlorinated 3-formylchromone derivative, the fused-ring system is slightly puckered [dihedral angle between the benzene and pyran rings $=3.66(10)^{\circ} \mathrm{]}$. The dihedral angle between the pyran ring and the formyl plane is $8.64(7)^{\circ}$. In the crystal, molecules are linked through $\pi-\pi$ stacking interactions [centroid-centroid distance between the benzene and pyran rings $=3.727$ (2) $\AA$ ], $\mathrm{C}-\mathrm{H} \cdots \mathrm{O}$ hydrogen bonds and short C. . O contacts [2.838 (4) A] . Halogen bonds between the formyl $\mathrm{O}$ atoms and the $\mathrm{Cl}$ atoms at the 7-position $\left[\mathrm{Cl} \cdots \mathrm{O}=2.984(3) \AA, \mathrm{C}-\mathrm{Cl} \cdots \mathrm{O}=170.83(12)^{\circ}\right.$ and $\mathrm{Cl} \cdots \mathrm{O}-\mathrm{C}=116.05(19)^{\circ}$ ] are also formed along the $a$ axis, resulting in helical structures constructed by $\mathrm{C}-\mathrm{H} \cdots \mathrm{O}$ hydrogen bonds and $\mathrm{Cl} \cdots \mathrm{O}$ halogen bonds along the $b$ axis. In addition, type II halogen-halogen contacts between the chlorine atoms at the 7- and 8-positions $[\mathrm{Cl} \cdots \mathrm{Cl}=3.519$ (2) $\AA$, C$\mathrm{Cl} \cdots \mathrm{Cl}=171.24(10)^{\circ}$ and $\left.88.74(11)^{\circ}\right]$ are observed.

\section{Chemical context}

Halogen bonding and halogen-halogen interactions have recently attracted much attention in medicinal chemistry, chemical biology, supramolecular chemistry and crystal engineering (Auffinger et al., 2004; Metrangolo et al., 2005; Wilcken et al., 2013; Sirimulla et al., 2013; Persch et al., 2015). Halogen bonding is defined as a net attractive interaction between an electrophilic region of a halogen atom in a molecule and a nucleophilic region of an atom in a molecule, and is characterized by a short contact between the two atoms. Halogen-halogen interactions are generally classified into two categories, type I $\left(\theta_{1}=\theta_{2}\right)$ and type II $\left(\theta_{1}=180, \theta_{2}=90\right)$ where $\theta_{1}$ and $\theta_{2}$ are the two $\mathrm{C}-\mathrm{Cl} \cdots \mathrm{Cl}$ angles. The type I contact is considered to be van der Waals, and the type II is halogen bonding, i.e., an electrostatic interaction (Mukherjee et al., 2014; Metrangolo et al., 2014).<smiles>O=Cc1coc2c(Cl)c(Cl)ccc2c1=O</smiles>

I have recently reported the crystal structures of chlorinated 3 -formylchromone derivatives 7-chloro-4-oxo-4H-chromene3-carbaldehyde (Ishikawa, 2014b), 8-chloro-4-oxo-4H-chromene-3-carbaldehyde (Ishikawa, 2014a) and 6,8-dichloro-4oxochromene-3-carbaldehyde (Ishikawa \& Motohashi, 2013). As for the monochlorinated 3-formylchromones, a type I van der Waals contact is observed in 7-chloro-4-oxo-4H-chro- 

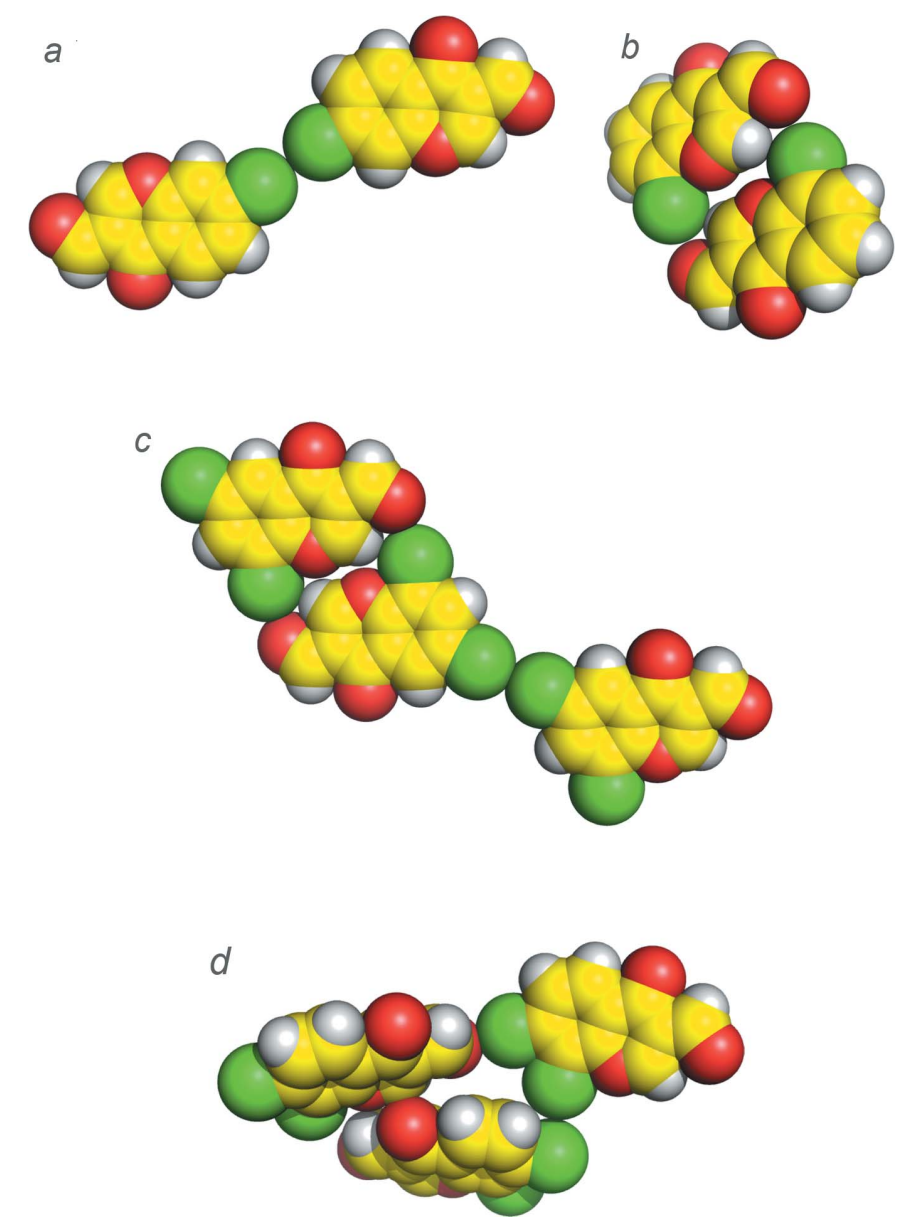

Figure 1

Sphere models of the crystal structures of (a) 7-chloro-4-oxo- $4 \mathrm{H}$ chromene-3-carbaldehyde (Ishikawa, 2014b), (b) 8-chloro-4-oxo-4Hchromene-3-carbaldehyde (Ishikawa, 2014a), (c) 6,8-dichloro-4-oxochromene-3-carbaldehyde (Ishikawa \& Motohashi, 2013) and (d) the title compound.

mene-3-carbaldehyde (Fig. 1a), and a van der Waals contact is observed between the formyl oxygen atom and the chlorine atom in 8-chloro-4-oxo-4H-chromene-3-carbaldehyde (Fig. 1b). On the other hand, as for the dichlorinated 3-formylchromone, halogen bonding between the formyl oxygen atom and the chlorine atom at the 8-position and a type I short halogen-halogen contact between the chlorine atoms at the 6-position are observed in 6,8-dichloro-4oxochromene-3-carbaldehyde (Fig. 1c). As part of our investigation into these types of chemical bonding, I herein report the crystal structure of a dichlorinated 3-formylchromone, 7,8-dichloro-4-oxo-4H-chromene-3-carbaldehyde. The main objective of this study is to reveal the interaction modes of the chlorine substituents of the title compound in the solid state.

\section{Structure commentary}

The molecular structure of the title compound is shown in Fig. 2. The fused-ring system is slightly puckered [dihedral angle between the benzene and pyran rings $\left.=3.66(10)^{\circ}\right]$. The
Table 1

Hydrogen-bond geometry $\left(\AA,^{\circ}\right)$.

\begin{tabular}{lllll}
\hline$D-\mathrm{H} \cdots A$ & $D-\mathrm{H}$ & $\mathrm{H} \cdots A$ & $D \cdots A$ & $D-\mathrm{H} \cdots A$ \\
\hline $\mathrm{C} 1-\mathrm{H} 1 \cdots \mathrm{O} 2^{\mathrm{i}}$ & 0.95 & 2.28 & $3.113(4)$ & $146(1)$ \\
$\mathrm{C} 4-\mathrm{H} 2 \cdots 3^{\text {ii }}$ & 0.95 & 2.47 & $3.398(4)$ & $167(1)$ \\
$\mathrm{C} 6-\mathrm{Cl} 1 \cdots{ }^{\mathrm{iii}}$ & $1.72(1)$ & $2.98(1)$ & $4.693(4)$ & $171(1)$ \\
$\mathrm{C} 10-\mathrm{O} 3 \cdots \mathrm{Cl} 1^{\text {iv }}$ & $1.21(1)$ & $2.98(1)$ & $3.678(4)$ & $116(1)$ \\
$\mathrm{C} 6-\mathrm{Cl} 1 \cdots \mathrm{Cl} 2^{\mathrm{v}}$ & $1.72(1)$ & $3.52(1)$ & $3.884(4)$ & $89(1)$ \\
$\mathrm{C} 7-\mathrm{C} 2 \cdots \mathrm{Cl}^{\mathrm{vi}}$ & $1.72(1)$ & $3.52(1)$ & $5.229(4)$ & $171(1)$ \\
\hline
\end{tabular}

Symmetry codes: (i) $-x+\frac{1}{2}, y-1, z-\frac{1}{2}$; (ii) $-x+\frac{1}{2}, y+1, z+\frac{1}{2}$; (iii) $x-\frac{1}{2},-y, z$; (iv) $x+\frac{1}{2},-y, z ;(\mathrm{v})-x,-y, z+\frac{1}{2} ;(\mathrm{vi})-x,-y, z-\frac{1}{2}$.

dihedral angle between the pyran ring and the formyl plane is $8.64(7)^{\circ}$.

\section{Supramolecular features}

In the crystal, the molecules are linked through $\pi-\pi$ stacking interactions between molecules related by translationsymmetry $^{\mathrm{i}}$ [centroid-centroid distance between the benzene and pyran rings of the $4 H$-chromene units $=3.727$ (2) $\AA$; symmetry code: (i) $x, y+1, z]$, and through $\mathrm{C}-\mathrm{H} \cdots \mathrm{O}$ hydrogen bonds that involve $\mathrm{C} 1 / \mathrm{O} 2$ and $\mathrm{C} 4 / \mathrm{O} 3$ atoms, as shown in Fig. 3 and listed in Table 1.

Halogen bonds are formed between the chlorine atom at the 7-position and the formyl oxygen atom ${ }^{\mathrm{ii}}$ along the $a$-axis direction $\left[\mathrm{Cl} 1 \cdots \mathrm{O} 3^{\mathrm{ii}}=2.984(3) \AA, \quad \mathrm{C} 6-\mathrm{Cl} 1 \cdots \mathrm{O} 3^{\mathrm{ii}}=\right.$ $170.83(12)^{\circ}, \mathrm{Cl} 1 \cdots \mathrm{O} 3^{\mathrm{ii}}-\mathrm{C} 10^{\mathrm{ii}}=116.05(19)^{\circ}$; symmetry code (ii) $\left.x-\frac{1}{2},-y, z\right]$, resulting in helical structures constructed by $\mathrm{C}-\mathrm{H} \cdots \mathrm{O}$ hydrogen bonds and $\mathrm{Cl}$.. O halogen bonds along the $b$-axis direction, as shown in Figs. 3 and 4. In addition, type II halogen-halogen contacts are observed between the chlorine atoms at the 7- and 8-position ${ }^{\text {iii }}\left[\mathrm{Cl} 1 \cdots \mathrm{Cl} 2^{\mathrm{iii}}=\right.$ 3.519 (2) $\AA, \mathrm{C} 7^{\mathrm{iii}}-\mathrm{Cl} 2^{\mathrm{iii}} \ldots \mathrm{Cl} 1=171.24(10)^{\circ}, \mathrm{C} 6-\mathrm{Cl} 1 \cdots \mathrm{Cl} 2^{\mathrm{iii}}=$ $88.74(11)^{\circ}$; symmetry code (iii) $\left.-x,-y, z+\frac{1}{2}\right]$, as shown in Fig. $1 d$. These electrostatic interactions around the chlorine atoms in the title compound are likely due to the cooperativity of the electron-withdrawing chlorine atoms at the 7- and 8-

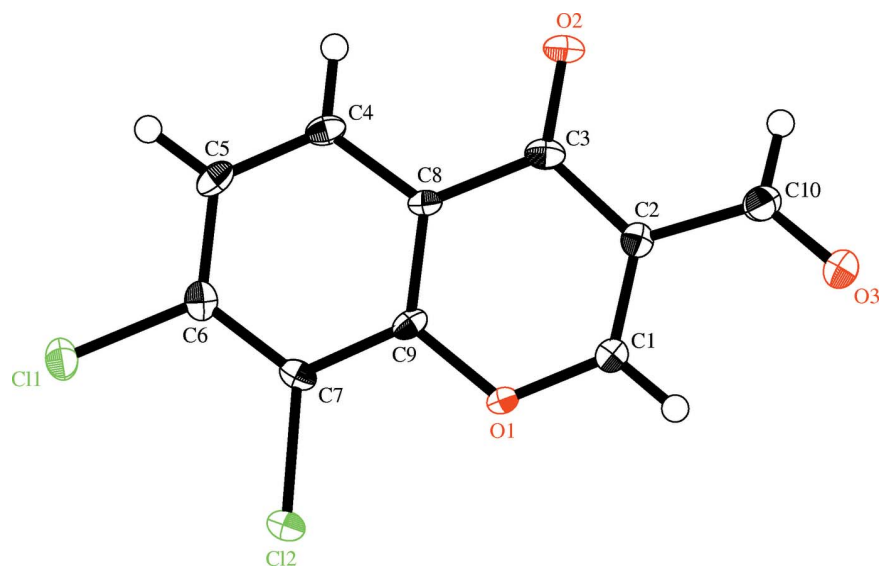

Figure 2

The molecular structure of the title compound, with displacement ellipsoids drawn at the $50 \%$ probability level. $\mathrm{H}$ atoms are shown as small spheres of arbitrary radius. 


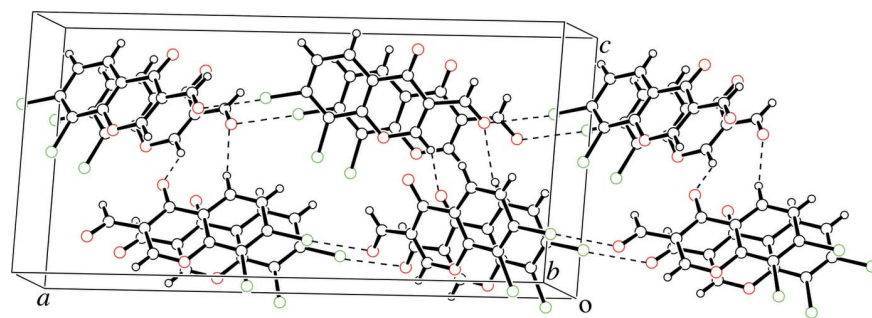

Figure 3

A packing view of the title compound. $\mathrm{C}-\mathrm{H} \cdots \mathrm{O}$ hydrogen bonds and $\mathrm{Cl} \cdots \mathrm{O}$ halogen bonds are represented as dashed lines.

positions. Thus, it is suggested that the chlorine atoms should make their $\sigma$-holes larger, and their electropositive regions contact the electronegative regions of the oxygen and chlorine atoms.

In addition to the $\mathrm{C}-\mathrm{H} \cdots \mathrm{O}$ hydrogen bonds, halogen bonds and type II halogen-halogen contacts, an unusually short contact is revealed between the $\alpha, \beta$-unsaturated carbonyl $\mathrm{O} 2$ and the $\mathrm{C}-\mathrm{H}$ group of $\mathrm{C}^{\text {iv }}$ [2.838 (4) $\AA$; symmetry code (iv) $-x+\frac{1}{2}, y, z+\frac{1}{2}$; Fig. 1d]. This interesting feature is possibly caused by a dipole-dipole interaction between the $\mathrm{O}$ atom and the $\mathrm{C}-\mathrm{H}$ group that is enhanced by the polarizing effect of the two chlorine atoms at the 7- and 8positions of the chromone ring. These observations should be helpful in understanding interactions of halogenated ligands with proteins, and thus valuable for rational drug design.

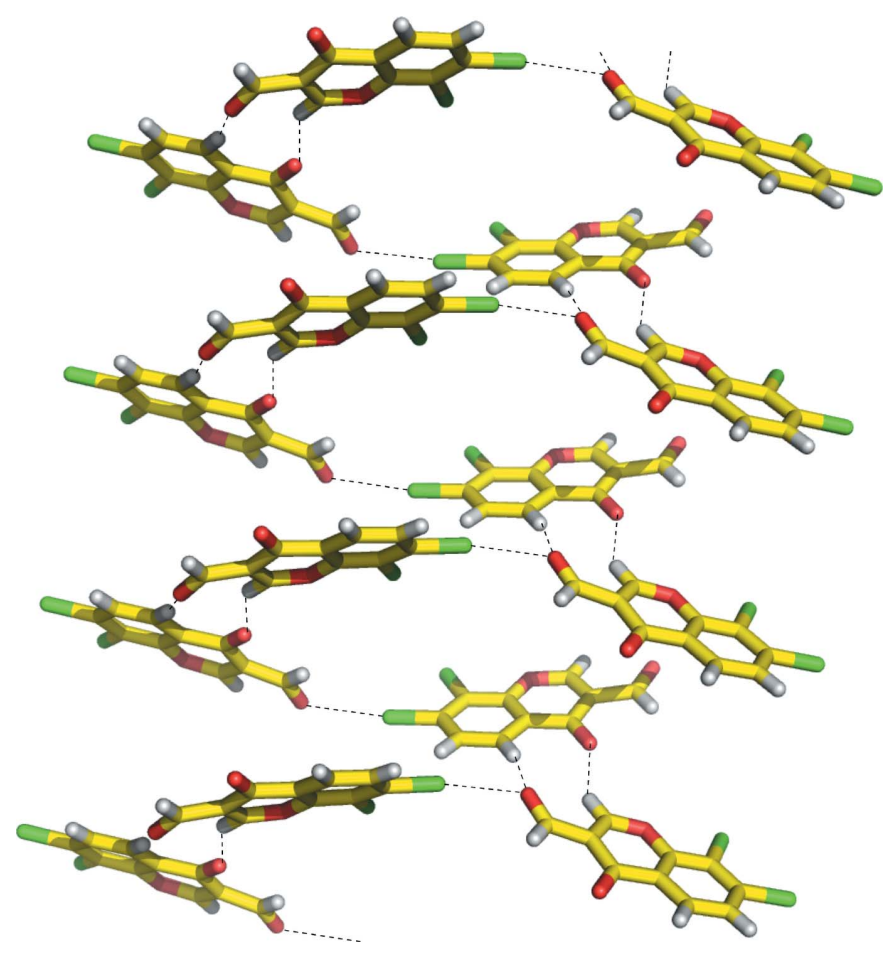

Figure 4

A helical structure constructed by $\mathrm{C}-\mathrm{H} \cdots \mathrm{O}$ hydrogen bonds and $\mathrm{Cl} \cdots \mathrm{O}$ halogen bonds in the crystal packing.
Table 2

Experimental details.

\begin{tabular}{|c|c|}
\hline \multicolumn{2}{|l|}{ Crystal data } \\
\hline Chemical formula & $\mathrm{C}_{10} \mathrm{H}_{4} \mathrm{Cl}_{2} \mathrm{O}_{3}$ \\
\hline$M_{\mathrm{r}}$ & 243.05 \\
\hline Crystal system, space group & Orthorhombic, $P c a 2_{1}$ \\
\hline Temperature $(\mathrm{K})$ & 100 \\
\hline$a, b, c(\AA)$ & $23.091(7), 3.7704(18), 10.729(5)$ \\
\hline$V\left(\AA^{3}\right)$ & $934.1(7)$ \\
\hline$Z$ & 4 \\
\hline Radiation type & Мо $K \alpha$ \\
\hline$\mu\left(\mathrm{mm}^{-1}\right)$ & 0.67 \\
\hline Crystal size $(\mathrm{mm})$ & $0.41 \times 0.12 \times 0.05$ \\
\hline \multicolumn{2}{|l|}{ Data collection } \\
\hline Diffractometer & Rigaku AFC-7R \\
\hline $\begin{array}{l}\text { No. of measured, independent and } \\
\text { observed }\left[F^{2}>2.0 \sigma\left(F^{2}\right)\right] \\
\text { reflections }\end{array}$ & $1571,1291,1155$ \\
\hline$R_{\text {int }}$ & 0.015 \\
\hline$(\sin \theta / \lambda)_{\max }\left(\AA^{-1}\right)$ & 0.650 \\
\hline \multicolumn{2}{|l|}{ Refinement } \\
\hline$R\left[F^{2}>2 \sigma\left(F^{2}\right)\right], w R\left(F^{2}\right), S$ & $0.025,0.057,1.05$ \\
\hline No. of reflections & 1291 \\
\hline No. of parameters & 136 \\
\hline No. of restraints & 1 \\
\hline $\mathrm{H}$-atom treatment & $\mathrm{H}$-atom parameters constrained \\
\hline$\Delta \rho_{\max }, \Delta \rho_{\min }\left(\mathrm{e} \AA^{-3}\right)$ & $0.24,-0.27$ \\
\hline Absolute structure & Flack (1983), 169 Friedel pairs \\
\hline Absolute structure parameter & $0.06(8)$ \\
\hline
\end{tabular}

Computer programs: WinAFC Diffractometer Control Software (Rigaku, 1999), SIR2008 (Burla et al., 2007), SHELXL97 (Sheldrick, 2008) and CrystalStructure (Rigaku, 2010).

\section{Synthesis and crystallization}

$3^{\prime}, 4^{\prime}$-Dichloro-2'-hydroxyacetophenone was prepared from 2,3-dichlorophenol by a Fries rearrangement reaction. To a solution of $3^{\prime}, 4^{\prime}$-dichloro-2' -hydroxyacetophenone $(5.9 \mathrm{mmol})$ in $N, N$-dimethylformamide $(20 \mathrm{ml})$ was added dropwise $\mathrm{POCl}_{3}(11.7 \mathrm{mmol})$ at $273 \mathrm{~K}$. After the mixture had been stirred for $14 \mathrm{~h}$ at room temperature, water $(100 \mathrm{ml})$ was added. The precipitates were collected, washed with water, and dried in vacuo (yield: $64 \%$ ). ${ }^{1} \mathrm{H} \mathrm{NMR} \mathrm{(400} \mathrm{MHz}, \mathrm{CDCl}_{3}$ ): $\delta=7.82(d, 1 \mathrm{H}, J=8.8 \mathrm{~Hz}), 8.08(d, 1 \mathrm{H}, J=8.8 \mathrm{~Hz}), 9.05(s$, $1 \mathrm{H}), 10.10(s, 1 \mathrm{H})$. Single crystals suitable for X-ray diffraction were obtained by slow evaporation of a tetrahydrofuran solution of the title compound at room temperature.

\section{Refinement}

Crystal data, data collection and structure refinement details are summarized in Table 2. The C-bound hydrogen atoms were placed in geometrical positions and refined using a riding model $\left[\mathrm{C}-\mathrm{H} 0.95 \AA\right.$ А,$\left.U_{\text {iso }}(\mathrm{H})=1.2 U_{\text {eq }}(\mathrm{C})\right]$.

\section{Acknowledgements}

The University of Shizuoka is acknowledged for instrumentation support.

\section{References}

Auffinger, P., Hays, F. A., Westhof, E. \& Ho, P. S. (2004). Proc. Natl Acad. Sci. USA, 101, 16789-16794. 
Burla, M. C., Caliandro, R., Camalli, M., Carrozzini, B., Cascarano, G. L., De Caro, L., Giacovazzo, C., Polidori, G., Siliqi, D. \& Spagna, R. (2007). J. Appl. Cryst. 40, 609-613.

Flack, H. D. (1983). Acta Cryst. A39, 876-881.

Ishikawa, Y. (2014a). Acta Cryst. E70, o743.

Ishikawa, Y. (2014b). Acta Cryst. E70, o831.

Ishikawa, Y. \& Motohashi, Y. (2013). Acta Cryst. E69, o1416.

Metrangolo, P., Neukirch, H., Pilati, T. \& Resnati, G. (2005). Acc. Chem. Res. 38, 386-395.

Metrangolo, P. \& Resnati, G. (2014). IUCrJ, 1, 5-7.

Mukherjee, A. \& Desiraju, G. R. (2014). IUCrJ, 1, 49-60.
Persch, E., Dumele, O. \& Diederich, F. (2015). Angew. Chem. Int. Ed.. 54, 3290-3327.

Rigaku (1999). WinAFC Diffractometer Control Software. Rigaku Corporation, Tokyo, Japan.

Rigaku (2010). CrystalStructure. Rigaku Corporation, Tokyo, Japan. Sheldrick, G. M. (2008). Acta Cryst. A64, 112-122.

Sirimulla, S., Bailey, J. B., Vegesna, R. \& Narayan, M. (2013). J. Chem. Inf. Model. 53, 2781-2791.

Wilcken, R., Zimmermann, M. O., Lange, A., Joerger, A. C. \& Boeckler, F. M. (2013). J. Med. Chem. 56, 1363-1388. 


\section{supporting information}

Acta Cryst. (2015). E71, 902-905 [https://doi.org/10.1107/S205698901501275X]

\section{Crystal structure of 7,8-dichloro-4-0xo-4H-chromene-3-carbaldehyde}

\section{Yoshinobu Ishikawa}

\section{Computing details}

Data collection: WinAFC Diffractometer Control Software (Rigaku, 1999); cell refinement: WinAFC Diffractometer Control Software (Rigaku, 1999); data reduction: WinAFC Diffractometer Control Software (Rigaku, 1999); program(s) used to solve structure: SIR2008 (Burla et al., 2007); program(s) used to refine structure: SHELXL97 (Sheldrick, 2008); molecular graphics: CrystalStructure (Rigaku, 2010); software used to prepare material for publication: CrystalStructure (Rigaku, 2010).

\section{7,8-Dichloro-4-oxo-4H-chromene-3-carbaldehyde}

\section{Crystal data}

$\mathrm{C}_{10} \mathrm{H}_{4} \mathrm{Cl}_{2} \mathrm{O}_{3}$

$M_{r}=243.05$

Orthorhombic, $P c a 2_{1}$

Hall symbol: P 2c -2ac

$a=23.091$ (7) $\AA$

$b=3.7704$ (18) $\AA$

$c=10.729(5) \AA$

$V=934.1(7) \AA^{3}$

$Z=4$

\section{Data collection}

Rigaku AFC-7R

diffractometer

$\omega$ scans

1571 measured reflections

1291 independent reflections

1155 reflections with $F^{2}>2.0 \sigma\left(F^{2}\right)$

$R_{\text {int }}=0.015$

\section{Refinement}

Refinement on $F^{2}$

$R\left[F^{2}>2 \sigma\left(F^{2}\right)\right]=0.025$

$w R\left(F^{2}\right)=0.057$

$S=1.05$

1291 reflections

136 parameters

1 restraint

Primary atom site location: structure-invariant direct methods

Secondary atom site location: difference Fourier map
$F(000)=488.00$

$D_{\mathrm{x}}=1.728 \mathrm{Mg} \mathrm{m}^{-3}$

Mo $K \alpha$ radiation, $\lambda=0.71069 \AA$

Cell parameters from 22 reflections

$\theta=15.8-17.3^{\circ}$

$\mu=0.67 \mathrm{~mm}^{-1}$

$T=100 \mathrm{~K}$

Plate, yellow

$0.41 \times 0.12 \times 0.05 \mathrm{~mm}$

$\theta_{\max }=27.5^{\circ}$

$h=0 \rightarrow 29$

$k=-4 \rightarrow 2$

$l=-7 \rightarrow 13$

3 standard reflections every 150 reflections

intensity decay: $1.1 \%$

Hydrogen site location: inferred from neighbouring sites

$\mathrm{H}$-atom parameters constrained

$w=1 /\left[\sigma^{2}\left(F_{\mathrm{o}}^{2}\right)+(0.0252 P)^{2}+0.2201 P\right]$ where $P=\left(F_{\mathrm{o}}^{2}+2 F_{\mathrm{c}}^{2}\right) / 3$

$(\Delta / \sigma)_{\max }<0.001$

$\Delta \rho_{\max }=0.24$ e $\AA^{-3}$

$\Delta \rho_{\min }=-0.27$ e $\AA^{-3}$

Absolute structure: Flack (1983), 169 Friedel pairs

Absolute structure parameter: 0.06 (8) 
Special details

Refinement. Refinement was performed using all reflections. The weighted $R$-factor $(w R)$ and goodness of fit $(S)$ are based on $F^{2} . R$-factor (gt) are based on $F$. The threshold expression of $F^{2}>2.0 \sigma\left(F^{2}\right)$ is used only for calculating $R$-factor (gt).

Fractional atomic coordinates and isotropic or equivalent isotropic displacement parameters $\left(\AA^{2}\right)$

\begin{tabular}{lllll}
\hline & $x$ & $y$ & $z$ & $U_{\text {iso }} / U_{\text {eq }}$ \\
\hline C11 & $-0.02754(3)$ & $0.3441(2)$ & $0.16617(7)$ & $0.02008(17)$ \\
C12 & $0.05650(3)$ & $-0.0003(3)$ & $-0.03533(8)$ & $0.01821(16)$ \\
O1 & $0.17307(8)$ & $-0.0690(6)$ & $0.04845(18)$ & $0.0137(5)$ \\
O2 & $0.24923(9)$ & $0.3154(5)$ & $0.36500(17)$ & $0.0179(5)$ \\
O3 & $0.34477(9)$ & $-0.3040(6)$ & $0.12579(19)$ & $0.0254(6)$ \\
C1 & $0.22935(12)$ & $-0.1270(8)$ & $0.0740(3)$ & $0.0136(7)$ \\
C2 & $0.25652(12)$ & $-0.0155(7)$ & $0.1782(3)$ & $0.0140(6)$ \\
C3 & $0.22568(13)$ & $0.1862(9)$ & $0.2724(3)$ & $0.0149(7)$ \\
C4 & $0.12566(13)$ & $0.3790(8)$ & $0.3362(3)$ & $0.0156(7)$ \\
C5 & $0.06756(13)$ & $0.4108(8)$ & $0.3106(3)$ & $0.0169(7)$ \\
C6 & $0.04536(12)$ & $0.2967(8)$ & $0.1961(3)$ & $0.0156(7)$ \\
C7 & $0.08124(12)$ & $0.1435(8)$ & $0.1075(3)$ & $0.0132(7)$ \\
C8 & $0.16288(12)$ & $0.2231(8)$ & $0.2485(3)$ & $0.0116(7)$ \\
C9 & $0.13996(12)$ & $0.1023(8)$ & $0.1362(3)$ & $0.0127(7)$ \\
C10 & $0.31843(13)$ & $-0.1099(9)$ & $0.1950(3)$ & $0.0195(7)$ \\
H1 & 0.2515 & -0.2554 & 0.0145 & $0.0163^{*}$ \\
H2 & 0.1406 & 0.4626 & 0.4133 & $0.0187^{*}$ \\
H3 & 0.0423 & 0.5109 & 0.3710 & $0.0203^{*}$ \\
H4 & 0.3385 & -0.0119 & 0.2642 & $0.0233^{*}$ \\
& & & & \\
\hline
\end{tabular}

Atomic displacement parameters $\left(\AA^{2}\right)$

\begin{tabular}{lllllll}
\hline & $U^{11}$ & $U^{22}$ & $U^{33}$ & $U^{12}$ & $U^{13}$ & $U^{23}$ \\
\hline C11 & $0.0144(3)$ & $0.0234(4)$ & $0.0224(4)$ & $0.0021(3)$ & $0.0007(4)$ & $0.0024(4)$ \\
C12 & $0.0181(3)$ & $0.0216(4)$ & $0.0149(3)$ & $-0.0005(4)$ & $-0.0043(4)$ & $-0.0019(4)$ \\
O1 & $0.0147(10)$ & $0.0162(12)$ & $0.0102(10)$ & $0.0015(9)$ & $-0.0001(9)$ & $-0.0031(9)$ \\
O2 & $0.0218(10)$ & $0.0199(11)$ & $0.0122(11)$ & $-0.0014(10)$ & $-0.0039(10)$ & $-0.0028(11)$ \\
O3 & $0.0199(12)$ & $0.0330(14)$ & $0.0233(13)$ & $0.0064(11)$ & $-0.0001(9)$ & $-0.0089(12)$ \\
C1 & $0.0154(13)$ & $0.0113(16)$ & $0.0140(15)$ & $0.0000(12)$ & $0.0013(13)$ & $0.0014(13)$ \\
C2 & $0.0152(14)$ & $0.0118(14)$ & $0.0148(15)$ & $0.0014(11)$ & $0.0005(13)$ & $0.0027(16)$ \\
C3 & $0.0214(15)$ & $0.0128(16)$ & $0.0106(14)$ & $-0.0016(13)$ & $-0.0019(13)$ & $0.0040(14)$ \\
C4 & $0.0224(15)$ & $0.0113(16)$ & $0.0130(16)$ & $-0.0017(13)$ & $0.0003(13)$ & $-0.0023(13)$ \\
C5 & $0.0209(15)$ & $0.0161(18)$ & $0.0137(15)$ & $-0.0012(14)$ & $0.0064(13)$ & $-0.0008(13)$ \\
C6 & $0.0148(13)$ & $0.0111(15)$ & $0.0208(18)$ & $0.0000(12)$ & $0.0011(12)$ & $0.0024(14)$ \\
C7 & $0.0152(14)$ & $0.0106(15)$ & $0.0139(15)$ & $-0.0045(13)$ & $-0.0033(12)$ & $0.0026(13)$ \\
C8 & $0.0149(14)$ & $0.0112(16)$ & $0.0087(14)$ & $-0.0025(12)$ & $-0.0002(12)$ & $-0.0002(12)$ \\
C9 & $0.0193(14)$ & $0.0093(14)$ & $0.0095(14)$ & $0.0016(12)$ & $0.0030(12)$ & $0.0016(12)$ \\
C10 & $0.0209(15)$ & $0.0208(17)$ & $0.0166(16)$ & $0.0003(14)$ & $0.0012(13)$ & $-0.0017(14)$ \\
& & & & & &
\end{tabular}


Geometric parameters $\left(\AA,{ }^{o}\right)$

\begin{tabular}{|c|c|c|c|}
\hline $\mathrm{C} 11-\mathrm{C} 6$ & $1.723(3)$ & $\mathrm{C} 4-\mathrm{C} 5$ & $1.375(5)$ \\
\hline $\mathrm{Cl} 2-\mathrm{C} 7$ & $1.723(3)$ & $\mathrm{C} 4-\mathrm{C} 8$ & $1.403(4)$ \\
\hline $\mathrm{O} 1-\mathrm{C} 1$ & $1.346(4)$ & $\mathrm{C} 5-\mathrm{C} 6$ & $1.399(5)$ \\
\hline $\mathrm{O} 1-\mathrm{C} 9$ & $1.374(4)$ & $\mathrm{C} 6-\mathrm{C} 7$ & $1.387(4)$ \\
\hline $\mathrm{O} 2-\mathrm{C} 3$ & $1.233(4)$ & $\mathrm{C} 7-\mathrm{C} 9$ & $1.399(4)$ \\
\hline $\mathrm{O} 3-\mathrm{C} 10$ & $1.207(4)$ & $\mathrm{C} 8-\mathrm{C} 9$ & $1.393(4)$ \\
\hline $\mathrm{C} 1-\mathrm{C} 2$ & $1.349(5)$ & $\mathrm{C} 1-\mathrm{H} 1$ & 0.950 \\
\hline $\mathrm{C} 2-\mathrm{C} 3$ & $1.452(4)$ & $\mathrm{C} 4-\mathrm{H} 2$ & 0.950 \\
\hline $\mathrm{C} 2-\mathrm{C} 10$ & $1.484(5)$ & $\mathrm{C} 5-\mathrm{H} 3$ & 0.950 \\
\hline $\mathrm{C} 3-\mathrm{C} 8$ & $1.479(4)$ & $\mathrm{C} 10-\mathrm{H} 4$ & 0.950 \\
\hline $\mathrm{Cl} 1 \cdots \mathrm{Cl} 2$ & $3.1821(13)$ & $\mathrm{C} 11 \cdots \mathrm{H} 3$ & 2.7981 \\
\hline $\mathrm{Cl} 2 \cdots \mathrm{O} 1$ & $2.850(3)$ & $\mathrm{O} 2 \cdots \mathrm{H} 2$ & 2.6203 \\
\hline $\mathrm{O} 1 \cdots \mathrm{C} 3$ & $2.860(4)$ & $\mathrm{O} 2 \cdots \mathrm{H} 4$ & 2.6344 \\
\hline $\mathrm{O} 2 \cdots \mathrm{C} 1$ & $3.570(4)$ & $\mathrm{O} 3 \cdots \mathrm{H} 1$ & 2.4697 \\
\hline $\mathrm{O} 2 \cdots \mathrm{C} 4$ & $2.880(4)$ & $\mathrm{C} 1 \cdots \mathrm{H} 4$ & 3.2719 \\
\hline $\mathrm{O} 2 \cdots \mathrm{C} 10$ & $2.907(4)$ & $\mathrm{C} 3 \cdots \mathrm{H} 1$ & 3.2841 \\
\hline $\mathrm{O} 3 \cdots \mathrm{C} 1$ & $2.803(4)$ & $\mathrm{C} 3 \cdots \mathrm{H} 2$ & 2.6882 \\
\hline $\mathrm{C} 1 \cdots \mathrm{C} 7$ & $3.587(4)$ & $\mathrm{C} 3 \cdots \mathrm{H} 4$ & 2.7113 \\
\hline $\mathrm{C} 1 \cdots \mathrm{C} 8$ & $2.758(5)$ & $\mathrm{C} 6 \cdots \mathrm{H} 2$ & 3.2648 \\
\hline $\mathrm{C} 2 \cdots \mathrm{C} 9$ & $2.765(4)$ & $\mathrm{C} 7 \cdots \mathrm{H} 3$ & 3.2742 \\
\hline $\mathrm{C} 4 \cdots \mathrm{C} 7$ & $2.803(4)$ & $\mathrm{C} 8 \cdots \mathrm{H} 3$ & 3.2642 \\
\hline $\mathrm{C} 5 \cdots \mathrm{C} 9$ & $2.766(4)$ & $\mathrm{C} 9 \cdots \mathrm{H} 1$ & 3.1865 \\
\hline $\mathrm{C} 6 \cdots \mathrm{C} 8$ & $2.785(4)$ & $\mathrm{C} 9 \cdots \mathrm{H} 2$ & 3.2686 \\
\hline $\mathrm{Cl} 1 \cdots \mathrm{Cl} 2^{\mathrm{i}}$ & $3.5191(17)$ & $\mathrm{C} 10 \cdots \mathrm{H} 1$ & 2.5378 \\
\hline $\mathrm{Cl} 1 \cdots \mathrm{O} 3^{\mathrm{ii}}$ & $2.984(3)$ & $\mathrm{H} 1 \cdots \mathrm{H} 4$ & 3.4723 \\
\hline $\mathrm{Cl} 2 \cdots \mathrm{Cl} 1^{\mathrm{iii}}$ & $3.5191(17)$ & $\mathrm{H} 2 \cdots \mathrm{H} 3$ & 2.3217 \\
\hline $\mathrm{O} 1 \cdots \mathrm{O} 2^{\text {iv }}$ & $3.533(3)$ & $\mathrm{Cl} 1 \cdots \mathrm{H}^{\mathrm{xi}}$ & 3.2315 \\
\hline $\mathrm{O} 1 \cdots \mathrm{O} 2^{\mathrm{v}}$ & $3.032(3)$ & $\mathrm{Cl} 1 \cdots \mathrm{H} 4^{\mathrm{ii}}$ & 3.4992 \\
\hline $\mathrm{O} 1 \cdots \mathrm{C} 8^{\mathrm{vi}}$ & $3.434(4)$ & $\mathrm{Cl} 2 \cdots \mathrm{H} 3^{\mathrm{iii}}$ & 3.1499 \\
\hline $\mathrm{O} 1 \cdots \mathrm{C} 9^{\mathrm{vi}}$ & $3.352(4)$ & $\mathrm{Cl} 2 \cdots \mathrm{H}^{\mathrm{xi}}$ & 3.1018 \\
\hline $\mathrm{O} 2 \cdots \mathrm{O} 1^{\mathrm{vii}}$ & $3.032(3)$ & $\mathrm{Cl} 2 \cdots \mathrm{H} 4^{v}$ & 3.2415 \\
\hline $\mathrm{O} 2 \cdots \mathrm{O} 1^{\text {viii }}$ & $3.533(3)$ & $\mathrm{O} 1 \cdots \mathrm{H} 1^{\mathrm{ix}}$ & 3.5807 \\
\hline $\mathrm{O} 2 \cdots \mathrm{C} 1^{\mathrm{vii}}$ & $2.838(4)$ & $\mathrm{O} 1 \cdots \mathrm{H} 4^{\mathrm{v}}$ & 3.0689 \\
\hline $\mathrm{O} 2 \cdots \mathrm{C} 1^{\text {viii }}$ & $3.113(4)$ & $\mathrm{O} 2 \cdots \mathrm{H} 1^{\mathrm{vii}}$ & 2.6840 \\
\hline $\mathrm{O} 2 \cdots \mathrm{C} 2^{\mathrm{ix}}$ & $3.227(4)$ & $\mathrm{O} 2 \cdots \mathrm{H} 1^{\mathrm{viii}}$ & 2.2789 \\
\hline $\mathrm{O} 2 \cdots \mathrm{C} 2^{\text {vii }}$ & $3.586(4)$ & $\mathrm{O} 2 \cdots \mathrm{H} 4^{\mathrm{ix}}$ & 3.4425 \\
\hline $\mathrm{O} 2 \cdots \mathrm{C} 3^{\mathrm{ix}}$ & $3.473(4)$ & $\mathrm{O} 3 \cdots \mathrm{H} 2^{\mathrm{iv}}$ & 2.4674 \\
\hline $\mathrm{O} 2 \cdots \mathrm{C} 10^{\mathrm{ix}}$ & $3.252(4)$ & $\mathrm{O} 3 \cdots \mathrm{H} 4^{\mathrm{vi}}$ & 3.0577 \\
\hline $\mathrm{O} 3 \cdots \mathrm{Cl1}^{\mathrm{x}}$ & $2.984(3)$ & $\mathrm{C} 1 \cdots \mathrm{H} 1^{\mathrm{ix}}$ & 3.3865 \\
\hline $\mathrm{O} 3 \cdots \mathrm{C} 2^{\mathrm{vi}}$ & $3.415(4)$ & $\mathrm{C} 2 \cdots \mathrm{H} 1^{\mathrm{ix}}$ & 3.3630 \\
\hline $\mathrm{O} 3 \cdots \mathrm{C} 4^{\mathrm{iv}}$ & $3.398(4)$ & $\mathrm{C} 3 \cdots \mathrm{H} 1^{\mathrm{ix}}$ & 3.5280 \\
\hline $\mathrm{O} 3 \cdots \mathrm{C} 10^{\mathrm{vi}}$ & $3.186(5)$ & $\mathrm{C} 3 \cdots \mathrm{H} 1^{\mathrm{vii}}$ & 3.1298 \\
\hline $\mathrm{C} 1 \cdots \mathrm{O} 2^{\mathrm{iv}}$ & $3.113(4)$ & $\mathrm{C} 3 \cdots \mathrm{H} 1^{\text {viii }}$ & 3.3849 \\
\hline $\mathrm{C} 1 \cdots \mathrm{O} 2^{\mathrm{v}}$ & $2.838(4)$ & $\mathrm{C} 4 \cdots \mathrm{H} 2^{\mathrm{vi}}$ & 3.5694 \\
\hline $\mathrm{C} 1 \cdots \mathrm{C}^{\mathrm{vi}}$ & $3.587(5)$ & $\mathrm{C} 5 \cdots \mathrm{H} 3^{\mathrm{vi}}$ & 3.5033 \\
\hline
\end{tabular}




\begin{tabular}{|c|c|c|c|}
\hline $\mathrm{C} 1 \cdots \mathrm{C} 3^{\mathrm{vi}}$ & $3.354(5)$ & $\mathrm{C} 6 \cdots \mathrm{H} 3^{\text {vi }}$ & 3.5082 \\
\hline $\mathrm{C} 1 \cdots \mathrm{C} 3^{\mathrm{v}}$ & $3.597(5)$ & $\mathrm{C} 8 \cdots \mathrm{H} 2^{\mathrm{vi}}$ & 3.4070 \\
\hline $\mathrm{C} 1 \cdots \mathrm{C} 8^{\mathrm{vi}}$ & $3.445(5)$ & $\mathrm{C} 10 \cdots \mathrm{H} 2^{\mathrm{iv}}$ & 3.5538 \\
\hline $\mathrm{C} 2 \cdots \mathrm{O} 2^{\mathrm{vi}}$ & $3.227(4)$ & $\mathrm{C} 10 \cdots \mathrm{H} 4^{\mathrm{vi}}$ & 3.5117 \\
\hline $\mathrm{C} 2 \cdots \mathrm{O} 2^{\mathrm{v}}$ & $3.586(4)$ & $\mathrm{H} 1 \cdots \mathrm{O} 1^{\mathrm{vi}}$ & 3.5807 \\
\hline $\mathrm{C} 2 \cdots \mathrm{O} 3^{\text {ix }}$ & $3.415(4)$ & $\mathrm{H} 1 \cdots \mathrm{O} 2^{\mathrm{iv}}$ & 2.2789 \\
\hline $\mathrm{C} 2 \cdots \mathrm{C} 1^{\text {ix }}$ & $3.587(5)$ & $\mathrm{H} 1 \cdots \mathrm{O} 2^{\mathrm{v}}$ & 2.6840 \\
\hline $\mathrm{C} 2 \cdots \mathrm{C} 3^{\mathrm{vi}}$ & $3.254(5)$ & $\mathrm{H} 1 \cdots \mathrm{C} 1^{\mathrm{vi}}$ & 3.3865 \\
\hline $\mathrm{C} 3 \cdots \mathrm{O} 2^{\mathrm{vi}}$ & $3.473(4)$ & $\mathrm{H} 1 \cdots \mathrm{C} 2^{\mathrm{vi}}$ & 3.3630 \\
\hline $\mathrm{C} 3 \cdots \mathrm{C} 1^{\mathrm{ix}}$ & $3.354(5)$ & $\mathrm{H} 1 \cdots \mathrm{C} 3^{\mathrm{vi}}$ & 3.5280 \\
\hline $\mathrm{C} 3 \cdots \mathrm{C} 1^{\mathrm{vii}}$ & $3.597(5)$ & $\mathrm{H} 1 \cdots \mathrm{C} 3^{\text {iv }}$ & 3.3849 \\
\hline $\mathrm{C} 3 \cdots \mathrm{C} 2^{\mathrm{ix}}$ & $3.254(5)$ & $\mathrm{H} 1 \cdots \mathrm{C} 3^{\mathrm{v}}$ & 3.1298 \\
\hline $\mathrm{C} 3 \cdots \mathrm{C} 10^{\text {ix }}$ & $3.510(5)$ & $\mathrm{H} 1 \cdots \mathrm{H} 2^{\text {iv }}$ & 2.9187 \\
\hline $\mathrm{C} 4 \cdots \mathrm{O} 3^{\text {viii }}$ & $3.398(4)$ & $\mathrm{H} 1 \cdots \mathrm{H} 4^{\mathrm{v}}$ & 3.5172 \\
\hline$C 4 \cdots C 8^{\text {ix }}$ & $3.428(5)$ & $\mathrm{H} 2 \cdots \mathrm{O}^{\text {viii }}$ & 2.4674 \\
\hline $\mathrm{C} 4 \cdots \mathrm{C} 9^{\mathrm{ix}}$ & $3.486(4)$ & $\mathrm{H} 2 \cdots \mathrm{C} 4^{\mathrm{ix}}$ & 3.5694 \\
\hline$C 5 \cdots C 6^{\text {ix }}$ & $3.596(5)$ & $\mathrm{H} 2 \cdots \mathrm{C} 8^{\mathrm{ix}}$ & 3.4070 \\
\hline$C 5 \cdots C 7^{\text {ix }}$ & $3.532(5)$ & $\mathrm{H} 2 \cdots \mathrm{C} 10^{\text {viii }}$ & 3.5538 \\
\hline $\mathrm{C} 6 \cdots 5^{\mathrm{vi}}$ & $3.596(5)$ & $\mathrm{H} 2 \cdots \mathrm{H} 1^{\text {viii }}$ & 2.9187 \\
\hline $\mathrm{C} 6 \cdots \mathrm{C} 7^{\mathrm{ix}}$ & $3.432(5)$ & $\mathrm{H} 3 \cdots \mathrm{Cl} 1^{\mathrm{xii}}$ & 3.2315 \\
\hline $\mathrm{C} 7 \cdots \mathrm{C}^{\mathrm{vi}}$ & $3.532(5)$ & $\mathrm{H} 3 \cdots \mathrm{Cl} 2^{\mathrm{i}}$ & 3.1499 \\
\hline $\mathrm{C} 7 \cdots \mathrm{C}^{\mathrm{vi}}$ & $3.432(5)$ & $\mathrm{H} 3 \cdots \mathrm{Cl} 2^{\mathrm{xii}}$ & 3.1018 \\
\hline $\mathrm{C} 8 \cdots \mathrm{O} 1^{\mathrm{ix}}$ & $3.434(4)$ & $\mathrm{H} 3 \cdots \mathrm{C} 5^{\text {ix }}$ & 3.5033 \\
\hline $\mathrm{C} 8 \cdots \mathrm{C} 1^{\mathrm{ix}}$ & $3.445(5)$ & $\mathrm{H} 3 \cdots \mathrm{C}^{\mathrm{ix}}$ & 3.5082 \\
\hline $\mathrm{C} 8 \cdots \mathrm{C} 4^{\mathrm{vi}}$ & $3.428(5)$ & $\mathrm{H} 4{ }^{\cdots} \mathrm{Cl}^{\mathrm{x}}$ & 3.4992 \\
\hline$C 8 \cdots C 9^{\text {ix }}$ & $3.567(5)$ & $\mathrm{H} 4 \cdots \mathrm{Cl} 2^{\mathrm{vii}}$ & 3.2415 \\
\hline $\mathrm{C} 9 \cdots \mathrm{O} 1^{\mathrm{ix}}$ & $3.352(4)$ & $\mathrm{H} 4 \cdots \mathrm{O} 1^{\mathrm{vii}}$ & 3.0689 \\
\hline $\mathrm{C} 9 \cdots \mathrm{C} 4^{\mathrm{vi}}$ & $3.486(4)$ & $\mathrm{H} 4 \cdots \mathrm{O} 2^{\mathrm{vi}}$ & 3.4425 \\
\hline $\mathrm{C} 9 \cdots \mathrm{C} 8^{\mathrm{vi}}$ & $3.567(5)$ & $\mathrm{H} 4 \cdots \mathrm{O} 3^{\mathrm{ix}}$ & 3.0577 \\
\hline $\mathrm{C} 10 \cdots \mathrm{O} 2^{\mathrm{vi}}$ & $3.252(4)$ & $\mathrm{H} 4 \cdots \mathrm{C} 10^{\mathrm{ix}}$ & 3.5117 \\
\hline $\mathrm{C} 10 \cdots \mathrm{O} 3^{\mathrm{ix}}$ & $3.186(5)$ & $\mathrm{H} 4 \cdots \mathrm{H} 1^{\mathrm{vii}}$ & 3.5172 \\
\hline $\mathrm{C} 10 \cdots \mathrm{C} 3^{\mathrm{vi}}$ & $3.510(5)$ & & \\
\hline $\mathrm{C} 1-\mathrm{O} 1-\mathrm{C} 9$ & $118.3(3)$ & $\mathrm{C} 3-\mathrm{C} 8-\mathrm{C} 4$ & $121.6(3)$ \\
\hline $\mathrm{O} 1-\mathrm{C} 1-\mathrm{C} 2$ & $124.5(3)$ & $\mathrm{C} 3-\mathrm{C} 8-\mathrm{C} 9$ & $119.5(3)$ \\
\hline $\mathrm{C} 1-\mathrm{C} 2-\mathrm{C} 3$ & $120.8(3)$ & $\mathrm{C} 4-\mathrm{C} 8-\mathrm{C} 9$ & $119.0(3)$ \\
\hline $\mathrm{C} 1-\mathrm{C} 2-\mathrm{C} 10$ & $118.3(3)$ & $\mathrm{O} 1-\mathrm{C} 9-\mathrm{C} 7$ & $116.2(3)$ \\
\hline $\mathrm{C} 3-\mathrm{C} 2-\mathrm{C} 10$ & $120.9(3)$ & $\mathrm{O} 1-\mathrm{C} 9-\mathrm{C} 8$ & $122.3(3)$ \\
\hline $\mathrm{O} 2-\mathrm{C} 3-\mathrm{C} 2$ & $123.5(3)$ & $\mathrm{C} 7-\mathrm{C} 9-\mathrm{C} 8$ & $121.5(3)$ \\
\hline $\mathrm{O} 2-\mathrm{C} 3-\mathrm{C} 8$ & $122.4(3)$ & $\mathrm{O} 3-\mathrm{C} 10-\mathrm{C} 2$ & $123.8(3)$ \\
\hline $\mathrm{C} 2-\mathrm{C} 3-\mathrm{C} 8$ & $114.2(3)$ & $\mathrm{O} 1-\mathrm{C} 1-\mathrm{H} 1$ & 117.736 \\
\hline $\mathrm{C} 5-\mathrm{C} 4-\mathrm{C} 8$ & $120.0(3)$ & $\mathrm{C} 2-\mathrm{C} 1-\mathrm{H} 1$ & 117.719 \\
\hline $\mathrm{C} 4-\mathrm{C} 5-\mathrm{C} 6$ & $120.4(3)$ & $\mathrm{C} 5-\mathrm{C} 4-\mathrm{H} 2$ & 119.983 \\
\hline $\mathrm{C} 11-\mathrm{C} 6-\mathrm{C} 5$ & $119.3(3)$ & $\mathrm{C} 8-\mathrm{C} 4-\mathrm{H} 2$ & 119.980 \\
\hline $\mathrm{C} 11-\mathrm{C} 6-\mathrm{C} 7$ & $120.0(3)$ & $\mathrm{C} 4-\mathrm{C} 5-\mathrm{H} 3$ & 119.801 \\
\hline $\mathrm{C} 5-\mathrm{C} 6-\mathrm{C} 7$ & $120.7(3)$ & $\mathrm{C} 6-\mathrm{C} 5-\mathrm{H} 3$ & 119.790 \\
\hline $\mathrm{Cl} 2-\mathrm{C} 7-\mathrm{C} 6$ & $122.8(3)$ & $\mathrm{O} 3-\mathrm{C} 10-\mathrm{H} 4$ & 118.100 \\
\hline $\mathrm{Cl} 2-\mathrm{C} 7-\mathrm{C} 9$ & $118.8(2)$ & $\mathrm{C} 2-\mathrm{C} 10-\mathrm{H} 4$ & 118.125 \\
\hline
\end{tabular}




$\begin{array}{ll}\mathrm{C} 6-\mathrm{C} 7-\mathrm{C} 9 & 118.3(3) \\ \mathrm{C} 1-\mathrm{O} 1-\mathrm{C} 9-\mathrm{C} 7 & -177.5(3) \\ \mathrm{C} 1-\mathrm{O} 1-\mathrm{C} 9-\mathrm{C} 8 & 1.3(4) \\ \mathrm{C} 9-\mathrm{O} 1-\mathrm{C} 1-\mathrm{C} 2 & -2.9(4) \\ \mathrm{C} 9-\mathrm{O} 1-\mathrm{C} 1-\mathrm{H} 1 & 177.1 \\ \mathrm{O} 1-\mathrm{C} 1-\mathrm{C} 2-\mathrm{C} 3 & -0.8(5) \\ \mathrm{O} 1-\mathrm{C} 1-\mathrm{C} 2-\mathrm{C} 10 & 178.1(3) \\ \mathrm{H} 1-\mathrm{C} 1-\mathrm{C} 2-\mathrm{C} 3 & 179.2 \\ \mathrm{H} 1-\mathrm{C} 1-\mathrm{C} 2-\mathrm{C} 10 & -1.9 \\ \mathrm{C} 1-\mathrm{C} 2-\mathrm{C} 3-\mathrm{O} 2 & -174.8(3) \\ \mathrm{C} 1-\mathrm{C} 2-\mathrm{C} 3-\mathrm{C} 8 & 5.5(4) \\ \mathrm{C} 1-\mathrm{C} 2-\mathrm{C} 10-\mathrm{O} 3 & -6.0(5) \\ \mathrm{C} 1-\mathrm{C} 2-\mathrm{C} 10-\mathrm{H} 4 & 173.9 \\ \mathrm{C} 3-\mathrm{C} 2-\mathrm{C} 10-\mathrm{O} 3 & 172.8(3) \\ \mathrm{C} 3-\mathrm{C} 2-\mathrm{C} 10-\mathrm{H} 4 & -7.2 \\ \mathrm{C} 10-\mathrm{C} 2-\mathrm{C} 3-\mathrm{O} 2 & 6.3(5) \\ \mathrm{C} 10-\mathrm{C} 2-\mathrm{C} 3-\mathrm{C} 8 & -173.4(3) \\ \mathrm{O} 2-\mathrm{C} 3-\mathrm{C} 8-\mathrm{C} 4 & -6.1(5) \\ \mathrm{O} 2-\mathrm{C} 3-\mathrm{C} 8-\mathrm{C} 9 & 173.5(3) \\ \mathrm{C} 2-\mathrm{C} 3-\mathrm{C} 8-\mathrm{C} 4 & 173.6(3) \\ \mathrm{C} 2-\mathrm{C} 3-\mathrm{C} 8-\mathrm{C} 9 & -6.8(4) \\ \mathrm{C} 5-\mathrm{C} 4-\mathrm{C} 8-\mathrm{C} 3 & 179.6(3) \\ \mathrm{C} 5-\mathrm{C} 4-\mathrm{C} 8-\mathrm{C} 9 & -0.0(4) \\ \end{array}$

$\begin{array}{ll}\mathrm{C} 8-\mathrm{C} 4-\mathrm{C} 5-\mathrm{C} 6 & -1.6(5) \\ \mathrm{C} 8-\mathrm{C} 4-\mathrm{C} 5-\mathrm{H} 3 & 178.4 \\ \mathrm{H} 2-\mathrm{C} 4-\mathrm{C} 5-\mathrm{C} 6 & -1.6 \\ \mathrm{H} 2-\mathrm{C} 4-\mathrm{C} 5-\mathrm{H} 3 & -0.4 \\ \mathrm{H} 2-\mathrm{C} 4-\mathrm{C} 8-\mathrm{C} 3 & 180.0 \\ \mathrm{H} 2-\mathrm{C} 4-\mathrm{C} 8-\mathrm{C} 9 & -179.4(3) \\ \mathrm{C} 4-\mathrm{C} 5-\mathrm{C} 6-\mathrm{C} 11 & 1.3(5) \\ \mathrm{C} 4-\mathrm{C} 5-\mathrm{C} 6-\mathrm{C} 7 & 0.6 \\ \mathrm{H} 3-\mathrm{C} 5-\mathrm{C} 6-\mathrm{C} 1 & -178.7 \\ \mathrm{H} 3-\mathrm{C} 5-\mathrm{C} 6-\mathrm{C} 7 & 1.0(4) \\ \mathrm{C} 11-\mathrm{C} 6-\mathrm{C} 7-\mathrm{C} 2 & -178.62(18) \\ \mathrm{C} 11-\mathrm{C} 6-\mathrm{C} 7-\mathrm{C} 9 & -179.7(3) \\ \mathrm{C} 5-\mathrm{C} 6-\mathrm{C} 7-\mathrm{C} 2 & 0.7(5) \\ \mathrm{C} 5-\mathrm{C} 6-\mathrm{C} 7-\mathrm{C} 9 & -3.2(4) \\ \mathrm{C} 12-\mathrm{C} 7-\mathrm{C} 9-\mathrm{O} 1 & 178.01(18) \\ \mathrm{C} 12-\mathrm{C} 7-\mathrm{C} 9-\mathrm{C} 8 & 176.4(3) \\ \mathrm{C} 6-\mathrm{C} 7-\mathrm{C} 9-\mathrm{O} 1 & -2.4(4) \\ \mathrm{C} 6-\mathrm{C} 7-\mathrm{C} 9-\mathrm{C} 8 & 3.7(4) \\ \mathrm{C} 3-\mathrm{C} 8-\mathrm{C} 9-\mathrm{O} 1 & -177.6(3) \\ \mathrm{C} 3-\mathrm{C} 8-\mathrm{C} 9-\mathrm{C} 7 & -176.7(3) \\ \mathrm{C} 4-\mathrm{C} 8-\mathrm{C} 9-\mathrm{O} 1 & 2.0(4) \\ \mathrm{C} 4-\mathrm{C} 8-\mathrm{C} 9-\mathrm{C} 7 & \end{array}$

Symmetry codes: (i) $-x,-y, z+1 / 2$; (ii) $x-1 / 2,-y, z$; (iii) $-x,-y, z-1 / 2$; (iv) $-x+1 / 2, y-1, z-1 / 2$; (v) $-x+1 / 2, y, z-1 / 2$; (vi) $x, y-1, z$; (vii) $-x+1 / 2, y, z+1 / 2$; (viii) $-x+1 / 2, y+1, z+1 / 2$; (ix) $x, y+1, z$; (x) $x+1 / 2,-y, z$; (xi) $-x,-y+1, z-1 / 2$; (xii) $-x,-y+1, z+1 / 2$.

Hydrogen-bond geometry $\left(\AA,{ }^{\circ}\right)$

\begin{tabular}{lllll}
\hline$D-\mathrm{H} \cdots A$ & $D-\mathrm{H}$ & $\mathrm{H} \cdots A$ & $D \cdots A$ & $D-\mathrm{H} \cdots A$ \\
\hline $\mathrm{C} 1-\mathrm{H} 1 \cdots \mathrm{O} 2^{\text {iv }}$ & 0.95 & 2.28 & $3.113(4)$ & $146(1)$ \\
$\mathrm{C} 4-\mathrm{H} 2 \cdots 3^{\text {viii }}$ & 0.95 & 2.47 & $3.398(4)$ & $167(1)$ \\
$\mathrm{C} 6-\mathrm{Cl1} \cdots \mathrm{O} 3^{\text {ii }}$ & $1.72(1)$ & $2.98(1)$ & $4.693(4)$ & $171(1)$ \\
$\mathrm{C} 10-\mathrm{O} 3 \cdots \mathrm{Cl1}^{\mathrm{x}}$ & $1.21(1)$ & $2.98(1)$ & $3.678(4)$ & $116(1)$ \\
$\mathrm{C} 6-\mathrm{Cl} 1 \cdots \mathrm{Cl} 2^{\mathrm{i}}$ & $1.72(1)$ & $3.52(1)$ & $3.884(4)$ & $89(1)$ \\
$\mathrm{C} 7-\mathrm{Cl} 2 \cdots \mathrm{Cl1} 1^{\mathrm{iii}}$ & $1.72(1)$ & $3.52(1)$ & $5.229(4)$ & $171(1)$
\end{tabular}

Symmetry codes: (i) $-x,-y, z+1 / 2$; (ii) $x-1 / 2,-y, z$; (iii) $-x,-y, z-1 / 2$; (iv) $-x+1 / 2, y-1, z-1 / 2$; (viii) $-x+1 / 2, y+1, z+1 / 2$; (x) $x+1 / 2,-y, z$. 